\title{
Sleep and Circadian Rhythm Disturbances in Eating Disorders
}

\author{
Sojeong Kim ${ }^{1,2}$ and Heon-Jeong Lee ${ }^{1,2}$ \\ 'Department of Psychiatry, Korea University College of Medicine, Seoul, Korea \\ ${ }^{2}$ Chronobiology Institute, Korea University, Seoul, Korea
}

\begin{abstract}
Sleep and circadian rhythm disturbances are common in patients with mental disorders. Past findings have suggested a close relationship between two critical behaviors of human, sleeping and eating. This review provides an overview of sleep and circadian rhythm problems that patients with anorexia nervosa, bulimia nervosa, binge eating disorder, and night eating syndrome experience. A line of studies included in this review confirmed that sleep and circadian rhythm are disturbed in patients with eating disorders. Current literature demonstrates that altered neuroendocrine circadian profiles may be associated with the feeding-fasting disruption. This review provides an insight into the relationship between circadian processes and eating disorder and emphasizes the importance of assessing circadian profiles in patients with eating disorder. In conclusion, we proposed future directions to better understand the sleep and circadian rhythm disruptions related to eating disorders.

Key Words: Sleep; Circadian rhythm; Eating disorders

Received: November 23, 2020 Accepted: November 30, 2020

Corresponding author: Heon-Jeong Lee, MD, PhD, Department of Psychiatry, Anam Hospital, Korea University College of Medicine, 73 Goryeodae-ro, Seongbuk-gu, Seoul 02841, Korea.

Tel: 82-2-920-6721, Fax: 82-2-927-9024, E-mail: leehjeong@korea.ac.kr

(c) This is an Open Access article distributed under the terms of the Creative Commons Attribution Non-Commercial License (https://creativecommons.org/licenses/bync/4.0) which permits unrestricted non-commercial use, distribution, and reproduction in any medium, provided the original work is properly cited.
\end{abstract}

\section{INTRODUCTION}

Eating and sleeping are two essential behaviors that humans engage in on a daily basis. Sleep disruption is frequently observed in patients with psychiatric disorders [1]. Sleep should be understood as a major phenotype of the circadian rhythm. Sleep/circadian rhythm disturbance is associated with increased sensitivity to a wide range of psychopathological conditions and is not only the result of difficulties in starting or sustaining sleep for individuals, but sleep/circadian systems are a result of complex interactions between many brain regions, neurotransmitters and modulatory hormone systems [2].

More recently, studies indicate sleep and circadian disturbance as a clinical marker closely associated with eating disorders. Some studies reported that patients with eating disorder who experience impaired sleep show more clinical symptoms including higher binge and vomiting frequency than patients without impaired sleep [3,4]. In a follow-up treatment study, Lombardo et al. [5] showed that patients with eating disorder who showed more sleep disturbances resulted in poorer treatment outcomes after six months than patients who did not. Also, a study showed that patients with eating disorders reported to be more of evening-types than healthy controls [6]. Along with treatment, patients with eating disorders tended to shift towards morning-type as symptom severity tended to decline. As such, past research highlights abnormalities in sleep and circadian pattern in eating disorder patients. In this review, we will investigate sleep and circadian dysregulation in four main groups: 1) anorexia nervosa (AN), 2) bulimia nervosa (BN), 3) binge eating disorder (BED), 4) night eating syndrome (NES). The current study decided to include NES because, recent studies started to propose the relationship between circadian misalignment and disordered night eating.

Since eating disorders are associated with high mortality rates, and comorbid sleep disturbances may result in more clinical symptoms and higher mortality rates [3], sleep in eating disorders should be thoroughly examined. The aim of this paper is not only to review the relationship between sleep/circadian rhythm and eating disorder but also to review hypothesized mechanisms of sleep and circadian disturbances in eating disorder. 


\section{ANOREXIA NERVOSA}

AN is characterized by inappropriate eating behavior, a pervasive pursuit of thinness, an intense fear of weight gain, and disturbed body image [7]. In general, researchers have subdivided AN into restricting type and bulimic type. Restrictors strive to keep themselves underweight by excessive dieting, fasting and exercising, and bulimics binge eat and engage in purging behaviors at times [8].

Sleep disturbance is generally known as a symptom and a risk factor of depression. Since AN often coexist with depression [9], past scholars have addressed the effect of mood symptoms in examining the sleep disturbances that eating disorder patients experience. Earlier studies using objective measures like electroencephalographic (EEG) reported that patients with AN showed greater number and longer awakenings from sleep than did the patients with depression alone $[10,11]$. Also, some studies have suggested that not all sleep parameters are associated with depression $[12,13]$. This is remarkable considering that impaired sleep is an established feature related to depression.

Regarding the features of impaired sleep, past studies showed that AN patients had more awakenings after sleep onset, reduced sleep efficiency, and reduced slow wave sleep $[12,14,15]$. However, some studies have failed to find significant differences in sleep parameters of AN patients and healthy controls except for differences in REM density [16] and self-reported sleep disturbances [17]. These conflicting observations may pertain to the heterogeneity of the samples. Patients diagnosed with AN may show marked difference in their eating patterns, depending on their key symptoms. A study indicated that patients diagnosed as AN can be subdivided into restrictors (55\%) and bulimics (45\%) [18]. As mentioned earlier, restrictors engage in fasting to keep their body weight low, whereas bulimic anorexics binge-eat at times. Therefore, differential symptoms of the subtypes should also be taken into consideration.

Several studies demonstrated that sleep disturbances in patients with AN may be associated with food deprivation and thus low body weight. An EEG study showed that AN patients at low body weight showed reduced sleep and more restlessness, especially decreased slow wave sleep [19]. Reduced slow wave sleep was correlated with body mass index (BMI). After weight gain, patients showed significant increase in sleep length and REM sleep [19]. In a study by Levy et al. [20], bulimic and anorexic patients did not show polysomnographic difference from those of healthy controls, but low weight anorexics showed less slow wave sleep. These studies may imply that food deprivation can cause decrease in slow wave sleep and total sleep length.

Studies on the relationship between reduced body weight and sleep disturbance in AN patients have implicated changes in a neuropeptide called orexin. Orexin is known to signal hunger as a response to starvation [21]. In rodents, starvation led to increased orexin level [22]. Increased orexin level is also known to promote wakefulness and arousal $[23,24]$. Also, administration of orexin receptor antagonists is known to promote sleep [25]. In many studies, treatment of insomnia symptoms using suvorexant, an orexin receptor antagonist has been reported to be generally effective [26-28]. Among women with AN, higher orexin level was associated with poorer self-reported sleep quality and sleep efficiency [29]. In addition, restricting type of AN patients showed decrease in orexin level after six months of treatment [30]. Taken together, studies point to a possibility of restricting anorexic patients experiencing sleep disturbances in relation to increased orexin level caused by starvation.

Furthermore, many researchers have suggested the importance of examining circadian hormone secretion in patients with AN. In a study by Galusca et al. [31], the circadian profile of an orexigenic peptide called $26 \mathrm{Fa}$ was significantly higher in AN patients especially during the afternoon and evening compared with healthy controls who showed higher values in the morning and abrupt decrease at noon. Patients with BN showed circadian profile of $26 \mathrm{Fa}$ comparable to those of healthy controls. 26Fa is reported to promote wakefulness in rodents and implied to be associated with arousal like orexin is [32]. Delayed 26Fa phase in AN patients may be related to the sleep problems that they experience. In addition, many studies have suggested that patients with AN show abnormality in circadian profiles of hormones that regulate food consumption like obestatin, ghrelin, and leptin [33-36]. Although these findings substantially confirm a link between circadian dysregulation and $\mathrm{AN}$, further studies need to examine the effect of abnormal circadian hormone secretion on symptoms of AN and vice versa.

\section{BULIMIA NERVOSA}

$\mathrm{BN}$ is characterized by recurrent binge eating episodes. Binge eating episodes are known as consumption of large amount of food with up to 10,000 calories taken during one episode. One important feature of binge episodes is that they happen with loss of control. To compensate for their binge eating, patients with $\mathrm{BN}$ engage in behaviors such as vomiting, laxative use, fasting, and excessive exercise. BN patients can be from normal weight to obese.

Compared to the volume of research in AN, sleep research in this diagnostic criterion is limited. Early studies suggest that sleep in $\mathrm{BN}$ is less disturbed than in AN [11]. For example, EEG data of $\mathrm{BN}$ patients showed that sleep pattern of $\mathrm{BN}$ patients were similar to healthy controls $[18,20,37]$. In a study by Latzer et al. [38], bulimic patients did not show significant differences in sleep duration, sleep latency, or other sleep quality measures but reported more complaints about their sleep than did the controls. Also, ambulatory actigraphy data revealed that $\mathrm{BN}$ patient's sleep-wake cycle is delayed by about 1 hour. This could be linked to their evening eating patterns. A recent study showed that BN patients reported more insomnia, daytime somnolence, and parasomnias assessed with self-reports and lengthened sleep latency, and reduction in sleep efficiency assessed with EEG [12]. 


\section{BINGE EATING DISORDER}

BED is characterized by recurrent binge eating episodes with loss of control. BED patients do not engage in compensatory behaviors such as vomiting, laxative misuse, fasting, and excessive exercise, which makes BED distinct from BN. Because they do not show inappropriate compensatory behaviors, they can be of normal weight but are often overweight or obese [39]. BED is more common than AN and BN [40].

Meta-analyses have suggested that obesity is associated with sleep disturbances including insomnia symptoms and short sleep duration [41-43]. Compared to the volume of literature on the relationship between obesity and sleep, sleep literature in BED is somewhat limited. Studies have shown that obese BED patients showed poorer sleep quality, increased sleep latency [44], and reduced sleep efficiency [45] compared to individuals with obesity alone. Also, a study by Trace et al. [46] showed that binge eating was positively associated with poor and shortened sleep and that this association remained significant even after controlling for obesity. However, there are studies that reported no significant difference in sleep parameters and circadian rhythm across obese individuals with and without BED $[47,48]$ and even healthy controls [49].

Some researchers have indicated that the pattern of binge eating is likely to be part of the mechanisms that work together to regulate sleep and circadian rhythm. First, a study found that sleep disturbances in patients who showed binge eating symptoms reported significantly more sleep disturbances than those who did not [4]. More recently, Yeh and Brown [50] reported that binge eating partly mediated the relationship of worse sleep quality to higher BMI whereas night eating mediated high BMI to worse sleep quality. This may imply that night eating and binge eating patterns of BED patients may contribute in the sleep and circadian disturbances they experience. Also, in a study by Harb et al. [51], individuals who showed more binge eating behaviors reported to be functioning better in the evening than in the morning by the Morningness-Eveningness Questionnaire (MEQ) scale, while there was no association between the MEQ score and BMI. Because binge eating is known to happen more in the evening than during the day $[52,53]$, evening chronotype may increase the probability of binge eating.

In understanding how binge eating pattern has its effect on sleep, researchers have proposed a crucial role of neuroendocrine that regulates appetite and satiety. Leptin, which signals satiety is one and ghrelin, which stimulates appetite when the body needs food is another. Experimental studies showed that sleep deprived group compared to usual sleep and extended sleep group showed significant decrease in leptin [54,55] and increase in ghrelin [54-56]. Decreased leptin level and increased ghrelin level prompt hunger and increased risk for obesity [57-59]. Taken together, it is plausible that sleep deprivation is associated with changes in feeding hormones and thus more food consumption and increased BMI.

In a recent study, Kenny et al. [60] showed that BED patients reported significantly greater insomnia symptoms than individuals without BED and that this relationship was partially and fully mediated by anxiety and depressive symptoms. This may imply that poor sleep that BED patients experience is related to mood. This is in line with studies that proposed that disordered eating is a maladaptive behavior associated with the difficulties in emotion regulation [61]. Mechanisms of relationship between sleep and BED are rather complicated and more to be studied.

\section{NIGHT EATING SYNDROME}

Sleep related eating disorder (SRED) and NES are two specific disorders of "eating disorders not otherwise specified (EDNOS)" that involve nocturnal eating. SRED patients experience recurrent episodes of night eating after sleep onset in a reduced state of alertness [8]. Some people show partial to no memory of their eating episodes, and others may recall their eating episodes [62]. Similarly, NES patients consume much of daily calories during the night. During their nocturnal awakenings, they feel a strong desire to intake food [63]. NES patients are usually aware of their night eating episodes [8]. A crucial difference that patients with SRED and NES experience is the level of awareness. In the current review, we focus on the diagnosis of NES.

Studies that employed nocturnal polysomnography reported that NES patients showed less stage two and three sleep, greater sleep disturbances assessed with Pittsburgh Sleep Quality Index compared to controls [64], more frequent nocturnal awakenings, and earlier first arousal during sleep [65]. Two other polysomnography studies that did not include a control group also reported that NES patients had frequent awakenings, which were associated with food consumption [66,67]. As such, studies have suggested that patients with NES show sleep disturbances in few different sleep parameters, especially more frequent nocturnal awakenings.

Laboratory and ambulatory studies have shown that NES patients consumed more amount of food during the evening and night compared to the controls $[63,65,68,69]$. Comparably, NES patients reported lower scores on the MEQ than did the controls, suggesting that they consider themselves to be functioning better during the evening than morning [11]. They also showed four hours delay in their eating patterns compared to the controls. Furthermore, Kandeger et al. [70] reported that higher the night eating scores, participants considered themselves to be more evening type by the MEQ score and that night eating scores had direct effect on MEQ scores. These findings imply that phase-delayed circadian rhythm may take part in the pathology of NES.

Past research has suggested that etiological mechanism of NES may be found in neuroendocrinal circadian rhythm. Birketvedt et al. [63] reported that NES patients showed decreased nocturnal rise in leptin and melatonin and elevated levels of plasma cortisol compared to the controls. As melatonin is known to induce and maintain sleep, decreased secretion of melatonin during the nights is associated with sleep disturbances that NES patients experience. Also, as rise in leptin secretion during the nights is 
known to regulate appetite, attenuated rise in leptin may contribute to nocturnal food consumption. Similarly, Goel et al. [69] also reported that both melatonin and leptin rhythms were phase delayed by an hour and that ghrelin, which is a stomach-derived appetite stimulant was phase advanced by 5.2 hours in patients with NES. Leptin and ghrelin are hormones regulated by putative peripheral oscillators that give signs for food consumption. Melatonin is a hormone regulated by the central timing system. Therefore, these findings may suggest that NES patients experience circadian dysregulation in both peripheral and central circadian timers. However, Allison et al. [68] did not find significant difference in values of melatonin and leptin between NES and control group. They suggested that the altered neuroendocrine circadian profiles of NES patients may not be the cause of altered food intake. There is a possibility that it could be a result and/or there could be an additional factor contributing to both food intake and hormonal patterns of NES.

\section{CIRCADIAN DYSREGULATION IN EATING DISORDERS: A HYPOTHESIS}

Eating is temporally coordinated over 24 hours of the circadian cycle. A network of circadian clocks controls when to rest and to be active and thus both eating and sleeping. Clocks that secure the circadian rhythm of food intake and sleep include a master clock that is entrained by light in the suprachiasmatic nuclei (SCN) of the hypothalamus and secondary clocks in the brain and peripheral organs. SCN, what is so called the master clock, is mainly entrained by light and thus is synchronized to the external environment. Closely affected by the light-dark cycle, SCN regulates the secondary clock such as food clock and engages in the feedingfasting cycle (Figure 1). In contrast to the SCN clock, which cannot be phase-shifted by the mealtime, food clock in the brain regions and peripheral tissues are reset to be entrained by mealtime. Metabolic hormones mediate the synchronization of the food clock to mealtime by promoting or inhibiting anticipation of food availability.

Among hormones that are related to food consumption, glucocorticoids, ghrelin, and glucagon are secreted before mealtime and are known as pre-feeding timers. Post-feeding timer hormones produced following a meal include insulin and leptin. First, glucocorticoids including cortisol and corticosterone are produced with a circadian rhythm and are increased in anticipation of the active phase. For example, during Ramadan when Muslims fast in the daytime and eat only in the nighttime, pre-prandial increase in plasma levels of cortisol is detected at dusk before the mealtime, while the usual peak of cortisol is detected at dawn as this is regulated by the SCN. As such, glucocorticoids act as a time-giver to synchronize behavioral and physiological functions to the expected mealtime. Ghrelin is another hormone that rises before and fall after a daytime meal and increases gradually overnight. This has led to the belief that ghrelin is related to meal initiation [71]. In rodents, ghrelin level showed a circadian rhythm both in light-

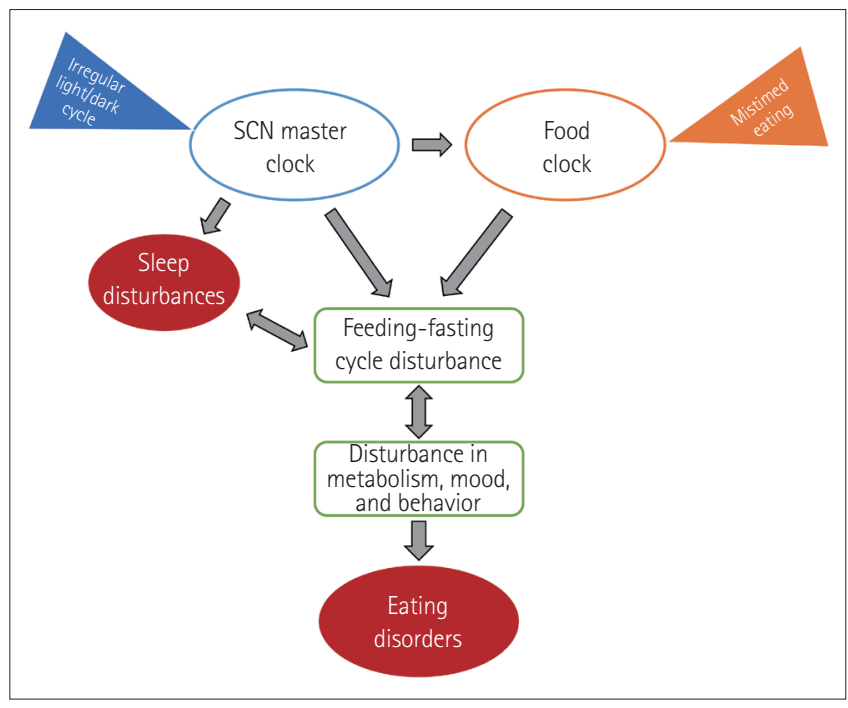

Figure 1. Regulation of eating by circadian clocks. A master clock in the suprachiasmatic nuclei ( $\mathrm{SCN}$ ) is synchronized by light and dark cycle. Food clock in the brain and peripheral organs is regulated by the master clock but can be synchronized by mealtime. SCN master clock and food clock both engage in the feeding-fasting cycle. Disruption of the feeding-fasting cycle can result in disturbance in metabolism, mood, and behavior. Underlying mechanisms of impaired eating and sleeping can be associated with circadian dysregulation.

dark cycles and in constant darkness with food provided ad libitum after 48 hours of food deprivation [72]. In humans, ghrelin administration is known to be associated with increased appetite $[71,58]$. These findings may indicate that ghrelin may promote anticipatory arousal and eating behavior. Glucagon, another pre-feeding timer hormone, is known to rise during restricted feeding [73]. A study confirmed that a protein-only diet elicits entrainment of circadian feeding clock in mice via glucagon [74]. Insulin, a postfeeding timer, is involved in resetting the peripheral clock that is associated with feeding behavior [75]. Leptin, another post-feeding timer, is known to take part in inhibiting food anticipatory activities, resulting in decreased food intake [76]. Although more is to be investigated, metabolic hormones can entrain the food clock to mealtime, and dysregulated circadian clocks can result in feeding-fasting disruption.

Circadian dysregulation and eating behaviors are reciprocally linked. On the one hand, altered timing of arousal-sleeping during the daytime for human - is associated with shifting of circadian oscillators in the brain and in the periphery. This dysregulation of circadian rhythm leads to alterations in the circadian rhythmicity of metabolic parameters and thus disruption in feeding behaviors. On the other hand, feeding time is also a synchronizing factor of circadian rhythm. Food consumption at usual resting time - nighttime for human — can desynchronize the circadian pattern and lead to circadian dysregulation. Also, mistimed eating has adverse effect on metabolic health such as increased risk of developing obesity and type 2 diabetes mellitus [77-79] and eating disorders especially NES; NES patients experience severe shifts in their food consumption towards later times of the day.

In summary, as the network of circadian clocks including a 
master clock in the SCN and food clock regulates the feeding and time of feeding, mistiming of the clocks can result in disturbances in feeding-fasting cycle, metabolism, mood, and behaviors, and thus in eating disorders (Figure 1). As mentioned earlier in the review, circadian misalignment is found to be associated with many symptoms of eating disorder. The current review suggests that circadian dysregulation is a potent mechanism underlying disordered eating behaviors.

\section{FUTURE DIRECTIONS}

Existing literature confirms that sleep and circadian rhythm are disturbed in patients with eating disorders. One exception was patients with $\mathrm{BN}$, who are reported to show comparable sleep parameters to those of healthy controls in many studies.

In the future, studies should be carried out to investigate the reason why patients with eating disorders often experience abnormalities in sleep patterns and circadian rhythm. Do symptoms of eating disorder transform sleep and circadian rhythm? Then parameters of sleep and circadian rhythm should recover with successful eating disorder treatment. Future studies should address specific symptoms of eating disorder including psychopathological correlates that have direct effect on sleep and circadian rhythm. Or do disrupted sleep and circadian rhythm affect symptoms of eating disorder? In this case, eating disorder interventions should emphasize treating disturbances in sleep and circadian rhythm. Here, more studies need to work on the role of impaired sleep and circadian rhythm as possible risk factors of eating disorder. Future studies would benefit from longitudinal design incorporating objective measures of both sleep and circadian rhythm on patients with eating disorder who experience sleep problems. Specific symptoms of eating disorders and objective parameters of sleep and circadian disturbances should be tracked along with the abnormalities in neuroendocrinal profiles to understand more thoroughly about the mechanism of the relationship of sleeping and eating.

\section{Acknowledgments}

None

\section{Conflicts of Interest}

The authors have no potential conflicts of interest to disclose.

\section{Author Contributions}

Conceptualization: Heon-Jeong Lee. Data curation: Sojeong Kim. Formal analysis: Sojeong Kim. Funding acquisition: Heon-Jeong Lee. Investigation: Sojeong Kim. Methodology: Sojeong Kim, Heon-Jeong Lee. Project administration: Heon-Jeong Lee. Supervision: Heon-Jeong Lee. Validation: Heon-Jeong Lee. Visualization: Sojeong Kim, Heon-Jeong Lee. Writing—original draft: Sojeong Kim. Writing_review \& editing: Sojeong Kim, Heon-Jeong Lee.

\section{ORCID iDs}

Sojeong Kim (1)

https://orcid.org/0000-0003-4970-4116

Heon-Jeong Lee (1)

https://orcid.org/0000-0002-9560-2383

\section{REFERENCES}

1. Short MA, Bartel K, Carskadon MA. Sleep and mental health in children and adolescents. In: Grandner MA, editor. Sleep and health. London: Academic Press, 2019, p. 435-445.

2. Pace-Schott EF, Hobson JA. The neurobiology of sleep: genetics, cellular physiology and subcortical networks. Nat Rev Neurosci 2002;3:591-605.

3. Arcelus J, Mitchell AJ, Wales J, Nielsen S. Mortality rates in patients with anorexia nervosa and other eating disorders: a meta-analysis of 36 studies. Arch Gen Psychiatry 2011;68:724-731.

4. Kim KR, Jung YC, Shin MY, Namkoong K, Kim JK, Lee JH. Sleep disturbance in women with eating disorder: prevalence and clinical characteristics. Psychiatry Res 2010;176:88-90.

5. Lombardo C, Battagliese G, Venezia C, Salvemini V. Persistence of poor sleep predicts the severity of the clinical condition after 6 months of standard treatment in patients with eating disorders. Eat Behav 2015;18:16-19.

6. Natale V, Ballardini D, Schumann R, Mencarelli C, Magelli V. Morningnesseveningness preference and eating disorders. Pers Individ Differ 2008;45:549553.

7. American Psychiatric Association. Diagnostic and statistical manual of mental disorders (DSM- ${ }^{\circledR}$ ). 5th ed. Arlington, VA: American Psychiatric Publishing; 2013.

8. Allison KC, Spaeth A, Hopkins CM. Sleep and eating disorders. Curr Psychiatry Rep 2016;18:92.

9. Godart N, Radon L, Curt F, Duclos J, Perdereau F, Lang F, et al. Mood disorders in eating disorder patients: prevalence and chronology of ONSET. J Affect Disord 2015;185:115-122.

10. Delvenne V, Kerkhofs M, Appelboom-Fondu J, Lucas F, Mendlewicz J. Sleep polygraphic variables in anorexia nervosa and depression: a comparative study in adolescents. J Affect Disord 1992;25:167-172.

11. Lundgren JD, O'Reardon JP, Allison KC, Spresser CD. Sleep and quality of life in eating disorders. In: Verster JC, Pandi-Perumal SR, Streiner DL, editors. Sleep and quality of life in clinical medicine. Totowa, NJ: Humana Press, 2008, p. 281-289.

12. Asaad Abdou T, Esawy HI, Abdel Razek Mohamed G, Hussein Ahmed H, Elhabiby MM, Khalil SA, et al. Sleep profile in anorexia and bulimia nervosa female patients. Sleep Med 2018;48:113-116.

13. Della Marca G, Farina B, Mennuni GF, Mazza S, Di Giannantonio M, Spadini V, et al. Microstructure of sleep in eating disorders: preliminary results. Eat Weight Disord 2004;9:77-80.

14. Neil JF, Merikangas JR, Foster FG, Merikangas KR, Spiker DG, Kupfer DJ. Waking and all-night sleep EEG's in anorexia nervosa. Clin Electroencephalogr 1980;11:9-15.

15. Nobili L, Baglietto MG, De Carli F, Savoini M, Schiavi G, Zanotto E, et al. A quantified analysis of sleep electroencephalography in anorectic adolescents. Biol Psychiatry 1999;45:771-775.

16. Lauer CJ, Krieg JC, Riemann D, Zulley J, Berger M. A polysomnographic study in young psychiatric inpatients: major depression, anorexia nervosa, bulimia nervosa. J Affect Disord 1990;18:235-245.

17. Latzer Y, Tzischinsky O, Epstein R. Sleep-wake monitoring in women suffering from anorexia nervosa. Eat Disord 2001;9:159-166.

18. Lauer C, Zulley J, Krieg JC, Riemann D, Berger M. EEG sleep and the cholinergic REM induction test in anorexic and bulimic patients. Psychiatry Res 1988;26:171-181.

19. Lacey JH, Crisp AH, Kalucy RS, Hartmann MK, Chien CN. Weight gain and the sleeping electroencephalogram: study of 10 patients with anorexia nervosa. Br Med J 1975;4:556-558.

20. Levy AB, Dixon KN, Schmidt H. REM and delta sleep in anorexia nervosa and bulimia. Psychiatry Res 1987;20:189-197.

21. Komaki G, Matsumoto Y, Nishikata H, Kawai K, Nozaki T, Takii M, et al. Orexin-A and leptin change inversely in fasting non-obese subjects. Eur J 
Endocrinol 2001;144:645-651.

22. Pankevich DE, Teegarden SL, Hedin AD, Jensen CL, Bale TL. Caloric restriction experience reprograms stress and orexigenic pathways and promotes binge eating. J Neurosci 2010;30:16399-16407.

23. Tsujino N, Sakurai T. Role of orexin in modulating arousal, feeding, and motivation. Front Behav Neurosci 2013;7:28.

24. Ohno K, Sakurai T. Orexin neuronal circuitry: role in the regulation of sleep and wakefulness. Front Neuroendocrinol 2008;29:70-87.

25. Brisbare-Roch C, Dingemanse J, Koberstein R, Hoever P, Aissaoui H, Flores $\mathrm{S}$, et al. Promotion of sleep by targeting the orexin system in rats, dogs and humans. Nat Med 2007;13:150-155.

26. Herring WJ, Snyder E, Budd K, Hutzelmann J, Snavely D, Liu K, et al. Orexin receptor antagonism for treatment of insomnia: a randomized clinical trial of suvorexant. Neurology 2012;79:2265-2274.

27. Herring WJ, Connor KM, Ivgy-May N, Snyder E, Liu K, Snavely DB, et al. Suvorexant in patients with insomnia: results from two 3-month randomized controlled clinical trials. Biol Psychiatry 2016;79:136-148.

28. Rhyne DN, Anderson SL. Suvorexant in insomnia: efficacy, safety and place in therapy. Ther Adv Drug Saf 2015;6:189-195.

29. Sauchelli S, Jiménez-Murcia S, Sánchez I, Riesco N, Custal N, FernándezGarcía JC, et al. Orexin and sleep quality in anorexia nervosa: clinical relevance and influence on treatment outcome. Psychoneuroendocrinology 2016;65:102-108.

30. Janas-Kozik M, Stachowicz M, Krupka-Matuszczyk I, Szymszal J, Krysta K, Janas A, et al. Plasma levels of leptin and orexin A in the restrictive type of anorexia nervosa. Regul Pept 2011;168:5-9.

31. Galusca B, Prévost G, Germain N, Dubuc I, Ling Y, Anouar Y, et al. Neuropeptide $\mathrm{Y}$ and $\alpha-\mathrm{MSH}$ circadian levels in two populations with low body weight: anorexia nervosa and constitutional thinness. PLoS One 2015;10: e0122040.

32. Takayasu S, Sakurai T, Iwasaki S, Teranishi H, Yamanaka A, Williams SC, et al. A neuropeptide ligand of the G protein-coupled receptor GPR103 regulates feeding, behavioral arousal, and blood pressure in mice. Proc Natl Acad Sci U S A 2006;103:7438-7443.

33. Germain N, Galusca B, Le Roux CW, Bossu C, Ghatei MA, Lang F, et al. Constitutional thinness and lean anorexia nervosa display opposite concentrations of peptide YY, glucagon-like peptide 1, ghrelin, and leptin. Am J Clin Nutr 2007;85:967-971

34. Germain N, Galusca B, Grouselle D, Frere D, Tolle V, Zizzari P, et al. Ghrelin/obestatin ratio in two populations with low bodyweight: constitutional thinness and anorexia nervosa. Psychoneuroendocrinology 2009;34:413419.

35. Germain N, Galusca B, Grouselle D, Frere D, Billard S, Epelbaum J, et al. Ghrelin and obestatin circadian levels differentiate bingeing-purging from restrictive anorexia nervosa. J Clin Endocrinol Metab 2010;95:3057-3062.

36. Misra M, Miller KK, Kuo K, Griffin K, Stewart V, Hunter E, et al. Secretory dynamics of ghrelin in adolescent girls with anorexia nervosa and healthy adolescents. Am J Physiol Endocrinol Metab 2005;289:E347-E356.

37. Walsh BT, Goetz R, Roose SP, Fingeroth S, Glassman AH. EEG-monitored sleep in anorexia nervosa and bulimia. Biol Psychiatry 1985;20:947-956.

38. Latzer Y, Tzischinsky O, Epstein R, Klein E, Peretz L. Naturalistic sleep monitoring in women suffering from bulimia nervosa. Int J Eat Disord 1999;26: 315-321.

39. de Zwaan M. Binge eating disorder and obesity. Int J Obes Relat Metab Disord 2001;25:S51-S55.

40. Hudson JI, Hiripi E, Pope HG Jr, Kessler RC. The prevalence and correlates of eating disorders in the National Comorbidity Survey Replication. Biol Psychiatry 2007;61:348-358.

41. Chan WS, Levsen MP, McCrae CS. A meta-analysis of associations between obesity and insomnia diagnosis and symptoms. Sleep Med Rev 2018;40:170182.

42. Wu Y, Zhai L, Zhang D. Sleep duration and obesity among adults: a metaanalysis of prospective studies. Sleep Med 2014;15:1456-1462.

43. Jean-Louis G, Youngstedt S, Grandner M, Williams NJ, Sarpong D, Zizi F, et al. Unequal burden of sleep-related obesity among black and white Americans. Sleep Health 2015;1:169-176.

44. Vardar E, Caliyurt O, Arikan E, Tuglu C. Sleep quality and psychopathological features in obese binge eaters. Stress Health 2004;20:35-41.

45. Tzischinsky O, Latzer Y. Sleep-wake cycles in obese children with and with- out binge-eating episodes. J Paediatr Child Health 2006;42:688-693.

46. Trace SE, Thornton LM, Runfola CD, Lichtenstein P, Pedersen NL, Bulik $\mathrm{CM}$. Sleep problems are associated with binge eating in women. Int J Eat Disord 2012;45:695-703

47. Tzischinsky O, Latzer Y, Epstein R, Tov N. Sleep-wake cycles in women with binge eating disorder. Int J Eat Disord 2000;27:43-48.

48. Roveda E, Montaruli A, Galasso L, Pesenti C, Bruno E, Pasanisi P, et al. Restactivity circadian rhythm and sleep quality in patients with binge eating disorder. Chronobiol Int 2018;35:198-207.

49. Filipova AA, Stoffel CL. The prevalence of binge eating disorder and its relationship to work and classroom productivity and activity impairment. J Am Coll Health 2016;64:349-361.

50. Yeh SS, Brown RF. Disordered eating partly mediates the relationship between poor sleep quality and high body mass index. Eat Behav 2014;15:291297.

51. Harb A, Levandovski R, Oliveira C, Caumo W, Allison KC, Stunkard A, et al. Night eating patterns and chronotypes: a correlation with binge eating behaviors. Psychiatry Res 2012;200:489-493.

52. Johnson WG, Schlundt DG, Barclay DR, Carr-Nangle RE, Engler LB. A naturalistic functional analysis of binge eating. Behav Ther 1995;26:101-118.

53. Mitchell JE, Pyle RL, Fletcher L. The topography of binge eating, vomiting and laxative abuse. Int J Eat Disord 1991;10:43-48.

54. Taheri S, Lin L, Austin D, Young T, Mignot E. Short sleep duration is associated with reduced leptin, elevated ghrelin, and increased body mass index. PLoS Med 2004;1:e62.

55. Spiegel K, Tasali E, Penev P, Van Cauter E. Brief communication: sleep curtailment in healthy young men is associated with decreased leptin levels, elevated ghrelin levels, and increased hunger and appetite. Ann Intern Med 2004; 141:846-850.

56. Schmid SM, Hallschmid M, Jauch-Chara K, Born J, Schultes B. A single night of sleep deprivation increases ghrelin levels and feelings of hunger in normalweight healthy men. J Sleep Res 2008;17:331-334.

57. Leibel RL. The role of leptin in the control of body weight. Nutr Rev 2002; 60(Suppl 10): S15-S19.

58. Wren AM, Seal LJ, Cohen MA, Brynes AE, Frost GS, Murphy KG, et al. Ghrelin enhances appetite and increases food intake in humans. J Clin Endocrinol Metab 2001;86:5992.

59. Korbonits M, Goldstone AP, Gueorguiev M, Grossman AB. Ghrelin--a hormone with multiple functions. Front Neuroendocrinol 2004;25:27-68.

60. Kenny TE, Van Wijk M, Singleton C, Carter JC. An examination of the relationship between binge eating disorder and insomnia symptoms. Eur Eat Disord Rev 2018;26:186-196.

61. Buckholdt KE, Parra GR, Anestis MD, Lavender JM, Jobe-Shields LE, Tull MT, et al. Emotion regulation difficulties and maladaptive behaviors: examination of deliberate self-harm, disordered eating, and substance misuse in two samples. Cogn Ther Res 2015;39:140-152.

62. Winkelman JW. Clinical and polysomnographic features of sleep-related eating disorder. J Clin Psychiatry 1998;59:14-19.

63. Birketvedt GS, Florholmen J, Sundsfjord J, Osterud B, Dinges D, Bilker W, et al. Behavioral and neuroendocrine characteristics of the night-eating syndrome. JAMA 1999;282:657-663.

64. Rogers NL, Dinges DF, Allison KC, Maislin G, Martino N, O'Reardon JP, et al. Assessment of sleep in women with night eating syndrome. Sleep 2006; 29:814-819.

65. O’Reardon JP, Ringel BL, Dinges DF, Allison KC, Rogers NL, Martino NS, et al. Circadian eating and sleeping patterns in the night eating syndrome. Obes Res 2004;12:1789-1796.

66. Spaggiari MC, Granella F, Parrino L, Marchesi C, Melli I, Terzano MG. Nocturnal eating syndrome in adults. Sleep 1994;17:339-344.

67. Manni R, Ratti MT, Tartara A. Nocturnal eating: prevalence and features in 120 insomniac referrals. Sleep 1997;20:734-738.

68. Allison KC, Lundgren JD, O’Reardon JP, Geliebter A, Gluck ME, Vinai P, et al. Proposed diagnostic criteria for night eating syndrome. Int J Eat Disord 2010;43:241-247.

69. Goel N, Stunkard AJ, Rogers NL, Van Dongen HP, Allison KC, O'Reardon JP, et al. Circadian rhythm profiles in women with night eating syndrome. J Biol Rhythms 2009;24:85-94.

70. Kandeger A, Egilmez U, Sayin AA, Selvi Y. The relationship between night eating symptoms and disordered eating attitudes via insomnia and chrono- 
type differences. Psychiatry Res 2018;268:354-357.

71. Cummings DE, Purnell JQ, Frayo RS, Schmidova K, Wisse BE, Weigle DS. A preprandial rise in plasma ghrelin levels suggests a role in meal initiation in humans. Diabetes 2001;50:1714-1719.

72. LeSauter J, Hoque N, Weintraub M, Pfaff DW, Silver R. Stomach ghrelin-secreting cells as food-entrainable circadian clocks. Proc Natl Acad Sci U S A 2009;106:13582-13587.

73. Mukherji A, Kobiita A, Chambon P. Shifting the feeding of mice to the rest phase creates metabolic alterations, which, on their own, shift the peripheral circadian clocks by 12 hours. Proc Natl Acad Sci U S A 2015;112:E6683E6690.

74. Ikeda Y, Kamagata M, Hirao M, Yasuda S, Iwami S, Sasaki H, et al. Glucagon and/or IGF-1 production regulates resetting of the liver circadian clock in response to a protein or amino acid-only diet. EBioMedicine 2018;28:210-224.
75. Yamajuku D, Inagaki T, Haruma T, Okubo S, Kataoka Y, Kobayashi S, et al. Real-time monitoring in three-dimensional hepatocytes reveals that insulin acts as a synchronizer for liver clock. Sci Rep 2012;2:439.

76. Kageyama H, Takenoya F, Hirako S, Wada N, Kintaka Y, Inoue S, et al. Neuronal circuits involving neuropeptide $\mathrm{Y}$ in hypothalamic arcuate nucleusmediated feeding regulation. Neuropeptides 2012;46:285-289.

77. Knutsson A, Kempe A. Shift work and diabetes--a systematic review. Chronobiol Int 2014;31:1146-1151.

78. Golombek DA, Casiraghi LP, Agostino PV, Paladino N, Duhart JM, Plano SA, et al. The times they're a-changing: effects of circadian desynchronization on physiology and disease. J Physiol Paris 2013;107:310-322.

79. Scheer FA, Hilton MF, Mantzoros CS, Shea SA. Adverse metabolic and cardiovascular consequences of circadian misalignment. Proc Natl Acad Sci U S A 2009;106:4453-4458. 